\section{New insights in the treatment of nontuberculous mycobacterial pulmonary disease}
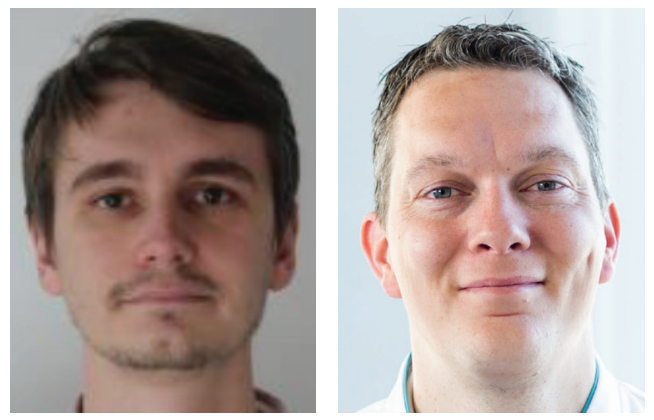

\author{
"For both Mycobacterium \\ avium complex and \\ M. abscessus, tedizolid and \\ clofazimine may help to \\ improve the efficacy of \\ treatment regimens."
}

Mike Marvin Ruth ${ }^{1} \&$ Jakko van Ingen ${ }^{*, 1}$

First draft submitted: 11 July 2017; Accepted for publication: 12 July 2017; Published online: 20 September 2017
Nontuberculous mycobacteria (NTM) are increasingly recognized as important opportunistic pathogens of humans. Chronic pulmonary infections are the most frequent diseases associated with NTM, particularly with Mycobacterium avium complex (MAC) and M. abscessus bacteria. This disease can manifest as a fibro-cavitary disease, mostly in patients with pre-existing pulmonary diseases or as a nodular/bronchiectatic disease, typically in elderly patients without underlying pulmonary diseases. In the latter group, subtle defects of mucociliairy clearance and T-cell immunity have been suggested to underlie susceptibility to NTM disease [1].

Currently recommended antibiotic treatment regimens are presented in Table 1. On average, 50 (M. abscessus) to $70 \% \mathrm{MAC}$ patients who tolerate recommended regimens achieve prolonged culture conversion [2]. This grave situation has recently led to a series of clinical and preclinical studies aiming to optimize antibiotic treatment of NTM pulmonary disease (NTM-PD).
Preclinical models have offered new leads to optimize antibiotic treatment. For both MAC and M. abscessus, tedizolid and clofazimine may help to improve the efficacy of treatment regimens. Tedizolid was recently shown to have MICs twoto four-fold lower than linezolid against both. Compared with linezolid, tedizolid has higher bioavailability, longer halflife, lower hematological and neurotoxicity rates, and it accumulates in the lung and macrophages [3]. The potential role of clofazimine became apparent in recent studies applying time-kill kinetic assays; clofazimine proved able to suppress the emergence of resistance to amikacin and clarithromycin, key drugs in NTM-PD treatment regimens [4].

For MAC bacteria, this rapid emergence of resistance was elegantly shown in hollow fiber pharmacodynamic models (HFM) by Schmalstieg et al., who showed that macrolide resistance in MAC is based on continuous increase of efflux pump expression followed by chromosomal mutation [5].
'Department of Medical Microbiology, Radboud University Medical Center, Nijmegen, The Netherlands

*Author for correspondence: Tel.: +31 24 3614356; jakko.vaningen@radboudumc.nl

\section{KEYWORDS}

- chronic pulmonary infections

- nontuberculous mycobacteria

- NTM 
"What we need are safe, tolerable and highly effective short-course regimens, preferable with oral antibiotics only."
Table 1. Currently recommended antibiotic treatment regimens.

\begin{tabular}{|ll|}
\hline Species & Recommended regimen \\
Mycobacterium avium complex & Rifampicin or rifabutin + ethambutol + macrolide \\
M. abscessus & $\begin{array}{l}\text { First phase: cefoxitin or imipenem + amikacin + macrolide } \\
\text { (+ tigecycline) } \\
\text { Second phase: macrolide + two oral drugs based on AST } \\
\text { (+ inhaled amikacin) }\end{array}$ \\
\hline Drugs in brackets are optional. & \\
AST: Antimicrobial susceptibility testing. & \\
\hline
\end{tabular}

From these data and Monte Carlo simulations, it appears that the macrolide antibiotic azithromycin, in its current recommended dose is ineffective in virtually all MAC patients [6]. The new antituberculosis drug bedaquiline proved bacteriostatic against $M$. avium in a mouse model, but it did not add to the activity of macrolides [7]. Perhaps, in synergistic combinations (like the bedaquiline-clofazimine combination already proven synergistic in mouse models of $M$. abscessus disease) [8] bedaquiline might still prove helpful in MAC pulmonary disease (MAC-PD) treatment.

For M. abscessus, preclinical studies reveal a dire picture which mirrors the clinical reality. Again using the HFM, Ferro et al. showed that moxifloxacin, which is currently recommended for M. abscessus treatment, is ineffective against it [9]. Also, the recommended initial regimen of amikacin-cefoxitin-clarithromycin fails to significantly reduce bacterial loads in the HFM and its limited activity is rapidly wiped out by the emergence of cefoxitin resistance [10]. This is in line with the earlier finding in a mouse model that cefoxitin alone, is as active as the full cefoxitin-amikacin-clarithromycin regimen [11]. More positive news was reported for tigecycline, which did prove effective against M. abscessus in HFM assays [12].

Animal models gave further valuable insights into the in vivo efficacy of $M$. abscessus treatment. Lefebvre et al. showed inhibition of $M$. abscessus $\beta$-lactamase by avibactam. This increased efficacy of imipenem, in vitro and in vivo, in a zebrafish model [13]. These findings give hope to empowering $\beta$-lactam antibiotics as a potent treatment option by coadministering avibactam. Bedaquiline also showed activity in the lung tissue of $M$. abscessus-infected mice [11]. The combination of bedaquiline and clofazimine showed a stronger synergistic effect; this combination therapy significantly lowered bacterial burden of $M$. abscessus in organs of interferongamma knockout $(\mathrm{GKO})$ mice $[8]$. These animal models mimic disseminated rather than pulmonary NTM disease and like the HFM findings for MAC and M. abscessus, they still await confirmation in clinical trials.

Some very influential clinical studies have come out in the past 5 years. One of the major changes in the MAC-PD field is the arrival of the first industry-sponsored randomized clinical trial. Within this trial, addition of liposomal amikacin by inhalation, to previously failing treatment regimens led to a significantly higher rate of culture conversion compared with placebo [14]. Bedaquiline has now also been tried in patients with MAC-PD, but microbiological outcomes were poor, with failures related to rapid emergence of bedaquiline resistance [15]. Applying bedaquiline in rifamycinfree treatments (e.g., with clofazimine instead) will increase bedaquiline exposure and might improve its efficacy.

Also, several case series have reported the outcomes of guideline-compliant MAC-PD treatment. In patients with nodular-bronchiectatic MAC-PD, groups from the USA and South Korea have shown that intermittent treatment with the rifamycin-ethambutol-macrolide regimen is highly successful, leading to prolonged culture conversion in $70-85 \%$ of the patients. Also, intermittent treatment was well tolerated and required fewer regimen modifications than daily treatment $[16,17]$. Owing to these patients' susceptibility to NTM-PD, recurrence rates remain high [17]. In a small fibro-cavitary MAC-PD cohort in The Netherlands, $57 \%$ of patients achieved prolonged culture conversion; addition of clofazimine and amikacin in patients with most severe disease yielded similar outcomes [18].

Clinical studies on M. abscessus treatment are fewer and consistently show poor outcomes, particularly for M. abscessus subspecies abscessus with its inducible macrolide resistance. The prognostic value of the detection of inducible or mutational macrolide resistance in the laboratory is now 
evident and this should be a standard practice [19]. One of the many questions regarding optimal antibiotic treatment of $M$. abscessus pulmonary disease is how many intravenous drugs are needed in the initiation phase of treatment; this has now been addressed in a small retrospective study, which concluded that in its cohort there was no evident benefit of two intravenous drugs during the initiation phase over only one [20]. The efficacy of clofazimine in $M$. abscessus treatment regimens is another question that has now been addressed for the first time, albeit again in a retrospective study only. In a cohort of 42 patients in South Korea, clofazimine-containing regimens lead to significant decreases in bacterial loads in sputum. Overall, complete culture conversion only occurred in $24 \%$ of the patients, but up to $40 \%$ of patients who received clofazimine as part of their initial regimen converted to negative cultures [21]. Both topics clearly warrant prospective clinical trials. In fact, prospective clinical trials of the currently recommended regimens have never been performed. The advent of new drugs and M. abscessus-specific clinical trials could deliver these as a byproduct.

But treatment outside of trials and reference clinics is a different world. Recent surveys have shown that adherence to the rifamycin-ethambutol-macrolide regimen for MAC-PD is very poor. The percentage of patients with MAC-PD that received $>6$ months of treatment with this regimen amounted to $44 \%$ in Japan, $21 \%$ in the USA and a shocking $10 \%$ in five European countries [22].
Despite significant progress, we are nowhere near where we need to be. We know better what (not) to expect of currently recommended regimens. We have a few exciting new antibiotics but we still have no new regimens. Regimens including either liposomal amikacin inhalation, bedaquiline or clofazimine seem effective against MAC-PD and tigecycline, tedizolid, bedaquiline-clofazimine, amikacin-clofazimine combinations and $\beta$-lactam antibiotics (with avibactam) appear effective against $M$. abscessus in preclinical studies. But how many and which companion drugs do we need, to maximize the impact of these drugs and combinations?

What we need are safe, tolerable and highly effective short-course regimens, preferable with oral antibiotics only. Finding these requires combined efforts by pharmaceutical companies and academia and an inclusive mentality leading to multicenter, global, pro and retrospective clinical studies, fueled by solid preclinical data.

\section{Financial \& competing interests disclosure}

$J$ van Ingen has served on advisory boards for Insmed, Janssen and Spero Therapeutics. He is supported by a grant from the Netherlands Organization for Scientific Research (NWOIZonMW grant Veni 016.176.024). The authors have no other relevant affiliations or financial involvement with any organization or entity with a financial interest in or financial conflict with the subject matter or materials discussed in the manuscript apart from those disclosed.

No writing assistance was utilized in the production of this manuscript.

\section{References}

1 Szymanski EP, Leung JM, Fowler CJ et al. Pulmonary nontuberculous mycobacterial infection. A multisystem, multigenic disease. Am. J. Respir. Crit. Care Med. 192(5), 618-628 (2015).

2 van Ingen J, Ferro BE, Hoefsloot W, Boeree MJ, van Soolingen D. Treatment of nontuberculous mycobacterial lung disease in HIV-negative patients: the evidence. Expert Rev. Anti. Infect. Ther. 11(10), 1065-1077 (2013).

3 Brown-Elliott BA, Wallace RJ Jr. In vitro susceptibility testing of tedizolid against nontuberculous mycobacteria. J. Clin. Microbiol. 55(6), 1747-1754 (2017).

4 Ferro BE, Meletiadis J, Wattenberg M et al. Clofazimine prevents the regrowth of Mycobacterium abscessus and Mycobacterium avium type strains exposed to amikacin and clarithromycin. Antimicrob. Agents Chemother. 60(2), 1097-1105 (2015).

5 Schmalstieg AM, Srivastava S, Belkaya S et al. The antibiotic resistance arrow of time: efflux pump induction is a general first step in the evolution of mycobacterial drug resistance. Antimicrob. Agents Chemother. 56(9), 4806-4815 (2012).

6 Deshpande D, Pasipanodya JG, Gumbo T. Azithromycin dose to maximize efficacy and suppress acquired drug resistance in pulmonary mycobacterium avium disease. Antimicrob. Agents Chemother. 60(4), 2157-2163 (2016).

7 Lounis N, Gevers T, van Den Berg J, Vranckx L, Andries K. ATP synthase inhibition of Mycobacterium avium is not bactericidal. Antimicrob. Agents Chemother. 53(11), 4927-4929 (2009).
8 Obregón-Henao A, Arnett KA, HenaoTamayo M et al. Susceptibility of Mycobacterium abscessus to antimycobacterial drugs in preclinical models. Antimicrob. Agents Chemother. 59(11), 6904-6912 (2015).

9 Ferro BE, Srivastava S, Deshpande D et al. Moxifloxacin's limited efficacy in the hollow-fiber model of Mycobacterium abscessus disease. Antimicrob. Agents Chemother. 60(6), 3779-3785 (2016).

10 Ferro BE, Srivastava S, Deshpande D et al. Failure of the amikacin, cefoxitin and clarithromycin combination regimen for treating pulmonary Mycobacterium abscessus infection. Antimicrob. Agents Chemother. 60(10), 6374-6376 (2016).

11 Lerat I, Cambau E, Roth Dit Bettoni R et al. In vivo evaluation of antibiotic activity against Mycobacterium abscessus. J. Infect. Dis. 209(6), 905-912 (2014). 
12 Ferro BE, Srivastava S, Deshpande D et al. Tigecycline is highly efficacious against Mycobacterium abscessus pulmonary disease. Antimicrob. Agents Chemother. 60(5), 2895-2900 (2016).

13 Lefebvre AL, Le Moigne V, Bernut A et al. Inhibition of the $\beta$-lactamase BlaMab by avibactam improves the in vitro and in vivo efficacy of imipenem against Mycobacterium abscessus. Antimicrob. Agents Chemother. 61(4), e02440-16 (2017).

14 Olivier KN, Griffith DE, Eagle G et al. Randomized trial of liposomal amikacin for inhalation in nontuberculous mycobacterial lung disease. Am. J. Respir. Crit. Care Med. 195(6), 814-823 (2017).

15 Alexander DC, Vasireddy R, Vasireddy S et al. The emergence of mmpT5 variants during bedaquiline treatment of
Mycobacterium intracellulare lung disease. J. Clin. Microbiol. 55(2), 574-584 (2017).

16 Jeong BH, Jeon K, Park HY et al. Intermittent antibiotic therapy for nodular bronchiectatic Mycobacterium avium complex lung disease. Am. J. Respir. Crit. Care Med. 191, 96-103 (2015).

17 Wallace RJ Jr, Brown-Elliott BA, McNulty S et al. Macrolide/azalide therapy for nodular/ bronchiectatic Mycobacterium avium complex lung disease. Chest 146(2), 276-282 (2014).

18 Zweijpfenning S, Kops S, MagisEscurra C, Boeree MJ, van Ingen J, Hoefsloot W. Treatment and outcome of nontuberculous mycobacterial pulmonary disease in a predominantly fibro-cavitary disease cohort. Resp. Med. doi:http://dx.doi. org/10.1016/j.rmed.2017.08.031 (2017) (Epub ahead of print).
19 Koh WJ, Jeon K, Lee NY et al. Clinical significance of differentiation of Mycobacterium massiliense from Mycobacterium abscessus. Am. J. Respir. Crit. Care Med. 183(3), 405-410 (2011).

20 Lyu J, Jang HJ, Song JW et al. Outcomes in patients with Mycobacterium abscessus pulmonary disease treated with long-term injectable drugs. Respir. Med. 105(5), 781-787 (2011).

21 Yang B, Jhun BW, Moon SM et al. Clofazimine-containing regimen for the treatment of Mycobacterium abscessus lung disease. Antimicrob. Agents Chemother. 61(6), e02052-16 (2017).

22 van Ingen J, Wagner D, Gallagher J et al. Poor adherence to management guidelines in nontuberculous mycobacterial pulmonary diseases. Eur. Respir. J. 49(2), pii:1601855 (2017). 